\title{
Percutaneous transcatheter biopsy for intracardiac mass diagnosis
}

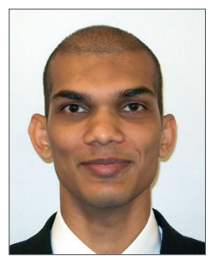

Gautam Reddy*, MD; Elad Maor, MD, PhD; Melanie C. Bois, MD; Krishnaswamy Chandrasekaran, MD; Charanjit S. Rihal, MD, MBA; Rick A. Nishimura, MD; David R. Holmes Jr, MD; Guy S. Reeder, MD; Joseph J. Maleszewski, MD; Kyle W. Klarich, MD

Mayo Clinic, Rochester, MN, USA

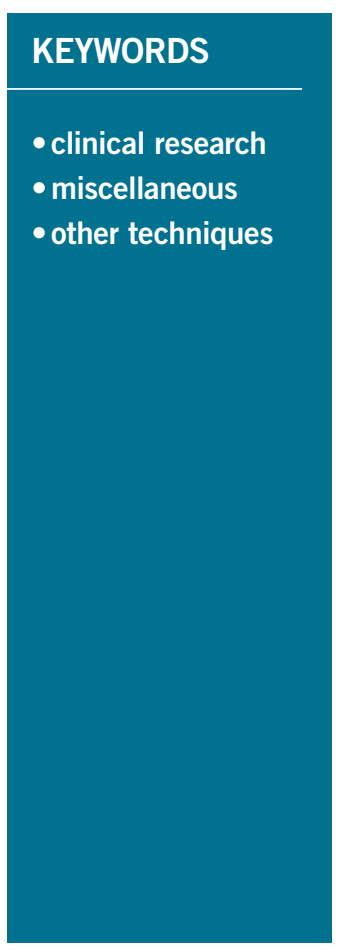

\begin{abstract}
Aims: The differential diagnosis of intracardiac masses (ICM) is wide. While imaging modalities can suggest a diagnosis, clinical decision making usually requires histopathologic diagnosis. The aim of this study was to describe the procedural technique, safety outcomes, diagnostic accuracy and clinical utility of percutaneous transcatheter biopsy (TCB) for histopathologic diagnosis of ICM. The records of all patients undergoing TCB of ICM at the Mayo Clinic catheterisation laboratories in Rochester, Minnesota, between 2002 and 2017 were retrieved and reviewed.
\end{abstract}

Methods and results: TCB of ICM to establish histopathologic diagnosis was performed in 29 patients. Masses were located in the right-sided chambers in $93 \%$ of cases. Echocardiographic guidance was used. Ventricular arrhythmias requiring immediate cardioversion occurred in $7 \%$ of patients. No other complications were noted. The average number of samples retrieved per procedure was $7 \pm 3.6$. A histopathologic diagnosis was made by TCB in $72 \%$ and altered clinical decision making in $52 \%$ of patients overall. Eleven patients (38\%) who would otherwise have required excisional biopsy were able to avoid cardiovascular surgery. Each additional biopsy sample was associated with an increase in the likelihood of making a histopathologic diagnosis (OR 1.74, 95\% CI: 1.05-2.87, $\mathrm{p}=0.032$ ).

Conclusions: Echo-guided percutaneous TCB of ICM provides an accurate diagnosis and alters clinical management in the majority of cases. The procedural complication rate is low. An increase in the number of samples retrieved markedly improves the ability to render a diagnosis. TCB may therefore be considered as a first-line approach for the histopathologic diagnosis of ICM.

*Corresponding author: The Mayo Clinic, 200 First Street SW, Rochester, MN 55905, USA.

E-mail: reddy.gautam@mayo.edu 


\section{Abbreviations}

$\begin{array}{ll}\text { CIED } & \text { cardiac implanted electronic device } \\ \text { CT } & \text { computed tomography } \\ \text { ICM } & \text { intracardiac mass } \\ \text { MR } & \text { magnetic resonance } \\ \text { PET } & \text { positron emission tomography } \\ \text { TCB } & \text { transcatheter biopsy } \\ \text { TEE } & \text { transoesophageal echocardiography } \\ \text { TTE } & \text { transthoracic echocardiography }\end{array}$

\section{Introduction}

The presence of an intracardiac mass (ICM) presents a significant diagnostic dilemma. The differential is wide and includes congenital, infectious, thrombotic, reactive, iatrogenic and neoplastic lesions ${ }^{1}$. Patients may be asymptomatic or present with constitutional symptoms, embolic phenomena and/or heart failure. These symptoms are non-specific and usually do not narrow the differential diagnosis ${ }^{2}$. The addition of cardiac imaging, with echocardiography $^{3}$, computed tomography $(\mathrm{CT})^{4}$ and magnetic resonance $(\mathrm{MR})^{5}$ has become standard practice for characterisation of $\mathrm{ICM}^{6}$. Identification of characteristic imaging features in the appropriate clinical setting can be virtually diagnostic for some lesions? In other cases, especially when malignancy is suspected, imaging may not be diagnostic. In these cases, critical therapeutic decisions may depend entirely on histopathologic diagnosis, which can also provide important prognostic information ${ }^{8}$. Excisional biopsy at the time of cardiovascular surgery is considered the definitive method to retrieve tissue from ICMs. However, this is associated with significant risks, including those related to general anaesthesia, median sternotomy, cardiopulmonary bypass and perioperative complications 9 . Percutaneous transcatheter biopsy (TCB) may present a minimally invasive diagnostic alternative in the diagnosis of ICM, especially for right-sided masses that are readily accessible to transcatheter devices. This procedure has been described in isolated case reports, but there are no large series in the literature to substantiate procedural technique, safety outcomes, diagnostic performance and clinical utility.

\section{Methods \\ STUDY POPULATION}

Approval was obtained from the Institutional Review Board. Medical records were screened for patients undergoing TCB of an ICM (2002-2017) at the Mayo Clinic in Rochester, Minnesota, USA. De-identified data were analysed for pre-specified procedural outcomes.

\section{PROCEDURE TECHNIQUE}

All procedures were performed in the catheterisation laboratory under local anaesthesia and conscious sedation. Venous access was obtained in the internal jugular vein $(59 \%)$ or common femoral vein (41\%). Jaw-type biopsy forceps (Cordis [Cardinal Health], Milpitas, CA, USA) were used to retrieve mass tissue. A transjugular liver biopsy needle (Argon Medical Devices, Plano, TX,
USA) was used when biopsy with forceps alone was deemed inadequate. Transseptal puncture and biopsy of a left atrial mass through a steerable Agilis ${ }^{\mathrm{TM}}$ sheath (St. Jude Medical, St. Paul, MN, USA) was performed in one case, and transthoracic Jamshidi ${ }^{\mathrm{TM}}$ needle biopsy (Beckton Dickinson and Company, Franklin Lakes, NJ, USA) of an anterior right ventricular mass was performed in another. Echocardiographic guidance with transthoracic or transoesophageal transducers was used in all cases to steer the biopsy device to the target lesion. Intracardiac echocardiography (ICE) was used when imaging windows were limited. When more than one ICM was present, the lesion that was most accessible was biopsied. When the mass was associated with cardiac implanted electronic device (CIED) leads, an attempt was made to perform the biopsy at a site distant from the leads. The number of biopsies obtained was left to the operator's discretion. Samples were preserved in saline and formalin. Coronary angiography and haemodynamic measurements were performed where indicated.

\section{OUTCOME DEFINITIONS}

The final histopathologic diagnosis was established in all cases. For isolated cardiac masses wherein surgical excision was not performed, the TCB tissue diagnosis was deemed final when histologic analysis showed pathologic tissue from a disease process consistent with the clinical setting and imaging findings. This was categorised as biopsy success. TCB was deemed non-diagnostic if only normal endomyocardial tissue or acute thrombotic material was retrieved, in a clinical setting inconsistent with these findings. This was categorised as biopsy failure. Surgical excision was performed for isolated cardiac masses when TCB was non-diagnostic or when the mass was symptomatic. In these cases, the diagnosis rendered from the excised material was considered final. For disease processes presenting with both cardiac and extracardiac masses, the final diagnosis was established via positive yield from TCB, cardiac surgical excisional biopsy, or biopsy material from non-cardiac sites.

\section{STATISTICAL ANALYSIS}

Analyses were performed using SPSS, Version 20 (IBM Corp., Armonk, NY, USA) and were pre-specified. Continuous variables were reported as mean and standard deviation. Discrete variables were reported as percentages. Binary logistic regression was used to evaluate the correlation between mass size, sample numbers and biopsy success. Sample number was used as a continuous and dichotomous variable in the models. Statistical significance was defined as a $\mathrm{p}$-value of $<0.05$.

\section{Results}

\section{PATIENT CHARACTERISTICS}

From 2002 to 2016, 29 patients underwent TCB for diagnosis of ICM (Table 1). The majority were women (55\%), with a mean age of $59 \pm 18$ years. A previous diagnosis of cancer had been made in $28 \%$, with $75 \%$ of these presumed to be in remission. Ninety percent $(90 \%)$ of patients were symptomatic, with dyspnoea $(72 \%)$, 
Table 1. Baseline characteristics of patients undergoing biopsy.

\begin{tabular}{|c|c|c|}
\hline \multicolumn{2}{|r|}{ Variable } & $\begin{array}{l}\text { Mean } \pm S D \text { or } \\
\text { percentage }\end{array}$ \\
\hline \multicolumn{2}{|l|}{ Sex (female) } & 55.2 \\
\hline \multicolumn{2}{|l|}{ Age (years) } & $59 \pm 17.6$ \\
\hline \multirow[t]{2}{*}{ Cancer history } & Diagnosis & 28 \\
\hline & Remission & 93 \\
\hline Electrocardiogram & non-specific & 59 \\
\hline \multirow[t]{2}{*}{ Cardiac device } & Pacemaker & 10.3 \\
\hline & Defibrillator & 3.4 \\
\hline \multirow[t]{2}{*}{ Mass dimensions } & Length $(\mathrm{cm})$ & $5.6 \pm 2.2$ \\
\hline & Width $(\mathrm{cm})$ & $3.9 \pm 1.6$ \\
\hline \multirow[t]{2}{*}{ Mass mobility } & Sessile & 82.7 \\
\hline & Pedunculated & 17.2 \\
\hline \multirow[t]{2}{*}{ Laboratory data } & Platelets $\left(10^{3} / \mu \mathrm{L}\right)$ & $249 \pm 94$ \\
\hline & INR level (ratio) & $1 \pm 0.19$ \\
\hline \multirow[t]{2}{*}{ Prior imaging } & Computed tomography & 75.9 \\
\hline & Magnetic resonance & 55.2 \\
\hline \multirow[t]{9}{*}{ Symptomatic status } & Asymptomatic & 10.3 \\
\hline & Dyspnoea & 72.4 \\
\hline & Fatigue & 65.5 \\
\hline & Arrhythmias & 10.3 \\
\hline & Chest pain & 17.2 \\
\hline & Heart murmur & 6.9 \\
\hline & IVC syndrome & 6.9 \\
\hline & SVC syndrome & 10.3 \\
\hline & Heart failure & 17.2 \\
\hline
\end{tabular}

INR: international normalised ratio; IVC: inferior vena cava;

SVC: superior vena cava

fatigue $(66 \%)$, and oedema (17\%) being most common. The mass was isolated to the heart in $59 \% ; 24 \%$ of patients had multiple ICMs (Figure 1). Right atrial involvement was noted in $66 \%$ of patients. Other locations included the right ventricle, superior vena cava, inferior vena cava, pulmonary artery and left-sided cardiac chambers. Ten percent $(10 \%)$ had masses associated with implanted pacemaker or defibrillator leads. The mass was sessile in $83 \%$ and pedunculated in $17 \%$. The mean size was $5.6 \pm 2.2 \mathrm{~cm}$. One mass was prolapsing across the tricuspid valve. A pericardial effusion was present prior to biopsy in $17 \%$ of patients.

\section{PROCEDURE DETAILS}

The procedures were performed by 14 different operators. The access site was an internal jugular vein in $59 \%$ of cases and the common femoral vein in the remainder (Table 2). The average venous sheath size was $8 \pm 1.1 \mathrm{Fr}$. The bioptome size was $7 \mathrm{Fr}$ in $69 \%$ and $9 \mathrm{Fr}$ in $31 \%$. Additional use of a side-cutting biopsy needle was noted in $10 \%$ of procedures (Figure 2). The average number of biopsy samples retrieved was $7 \pm 3.6$. Univariate binary logistic regression with sample number as a continuous variable showed that each additional sample was associated

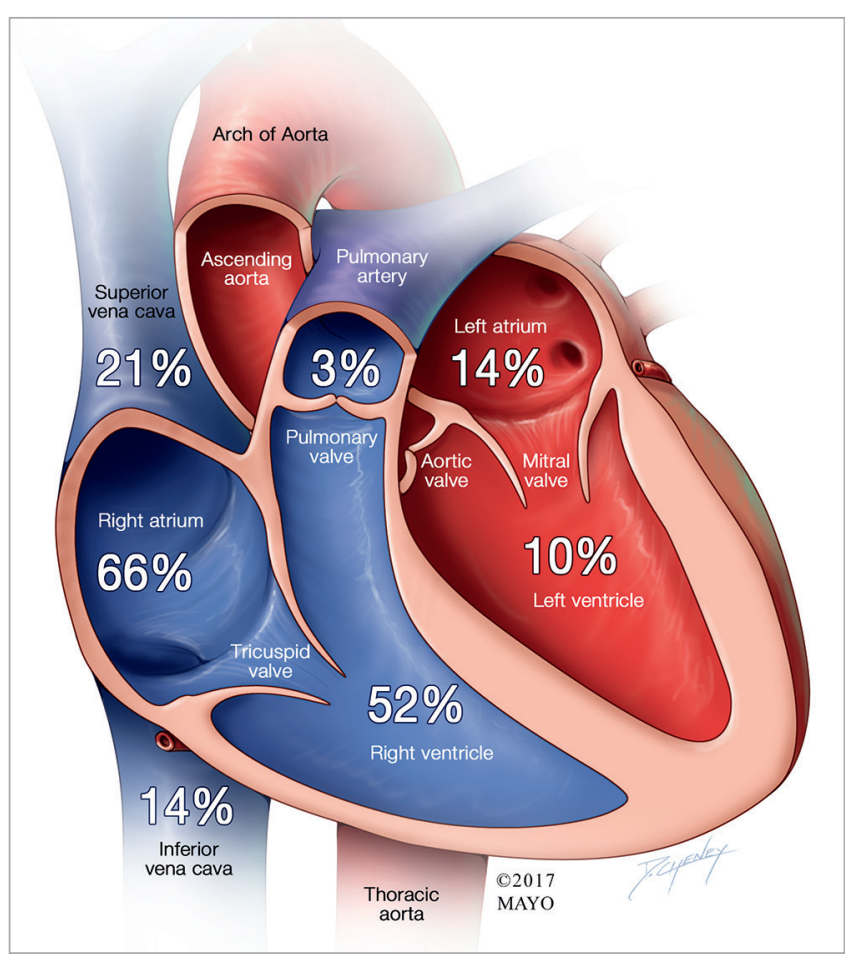

Figure 1. Cardiac chambers involved by masses targeted for biopsy. Cutaway diagram of the heart showing the anatomic location of the intracardiac masses in this series. Multiple locations are coded in each case for singular masses extending into multiple chambers and for multiple masses in different locations.

with a significant increase in the likelihood of biopsy success (OR 1.74, 95\% CI: 1.05-2.87, p=0.032). Using the number of samples as a dichotomous variable showed that procedures during which more than seven samples (median) were retrieved were 16 times more likely to result in biopsy success (OR 16.33, 95\%

Table 2. Transcatheter biopsy procedural characteristics.

\begin{tabular}{|l|l|c|}
\hline \multicolumn{2}{|c|}{ Variable } & MeantSD or percentage \\
\hline \multirow{2}{*}{ Access route } & Internal jugular & 59 \\
\cline { 2 - 3 } & Common femoral & 41 \\
\cline { 2 - 3 } & Transseptal puncture & 3.5 \\
\hline French size & Sheath & $8 \pm 1.1$ \\
\cline { 2 - 3 } & Bioptome & $8 \pm 0.9$ \\
\hline Alternative devices & 13.8 \\
\hline Cutting needle & 10.3 \\
\hline Jamshidi needle & 3.5 \\
\hline Biopsy samples & $7 \pm 3.5$ \\
\hline Echocardiography & 100 \\
\hline Transthoracic & 82.8 \\
\hline Transoesophageal & 17.2 \\
\hline Intracardiac & 3.5 \\
\hline Complications & 6.9 \\
\hline Ventricular tachycardia & 6.9 \\
\hline
\end{tabular}




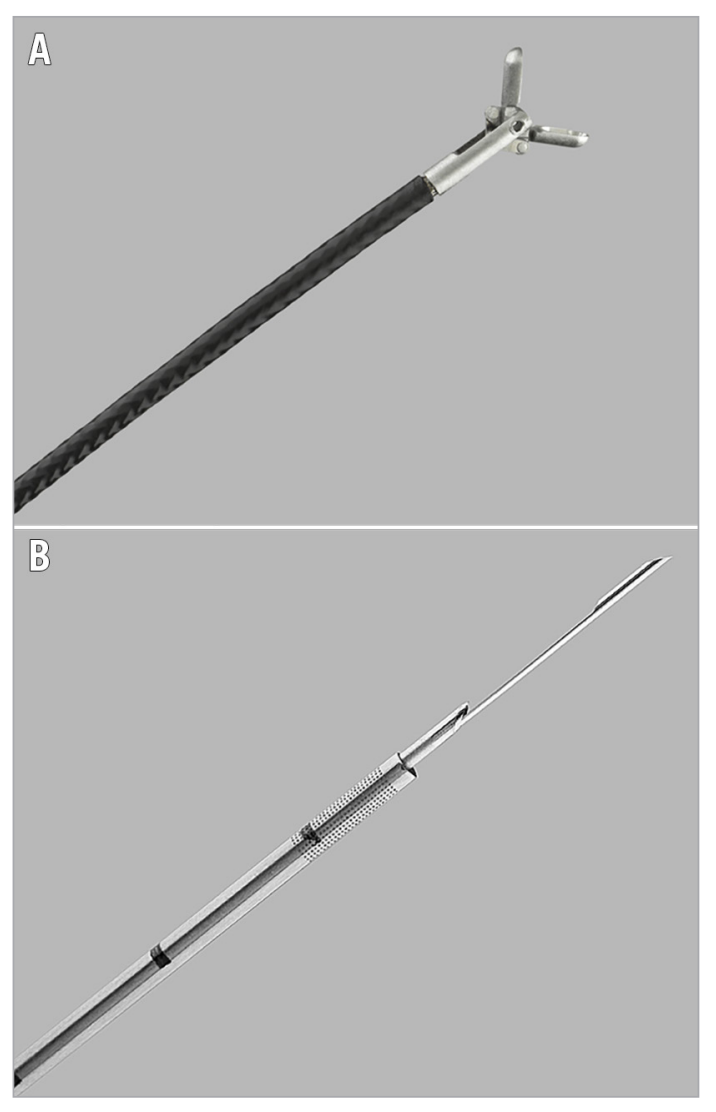

Figure 2. Images of jaw-type bioptome and side-cutting needle. A) Flexible endomyocardial bioptome with articulated jaws. A hand-operated lever on the opposite end controls opening. B) Flexible side-cutting needle normally used for transjugular liver biopsies. This can be adapted for percutaneous intracardiac biopsies.

CI: 1.63-163, $\mathrm{p}=0.017$ ) (Figure 3). Uniyariate models showed no association between biopsy success, mass area, minimal diameter or maximal diameter ( $p>0.10$ for all). Echo guidance was used in $100 \%$ (transthoracic [TTE] $83 \%$ and transoesophageal [TEE] $17 \%$ ). ICE was used in one case because of poor visualisation with TTE and TEE imaging. Two patients (7\%) had complications ventricular tachycardia developed upon bioptome contact with the ventricle and required immediate cardioversion. No other complications were noted. The four patients (13.7\%) with CIEDs showed no signs of lead damage.

\section{ALTERNATIVE ROUTES}

One patient presented with a left atrial mass and was deemed to have prohibitive surgical risk. A transvenous transseptal puncture was performed and a steerable Agilis sheath was used to direct a bioptome towards the mass. A positive biopsy result for epithelioid leiomyosarcoma was obtained (Figure 4). No embolic complications were noted. One patient had an infiltrative transmural mass in the wall of the right ventricle. Tissue yield with a jawstype bioptome via the transvenous route was deemed inadequate.
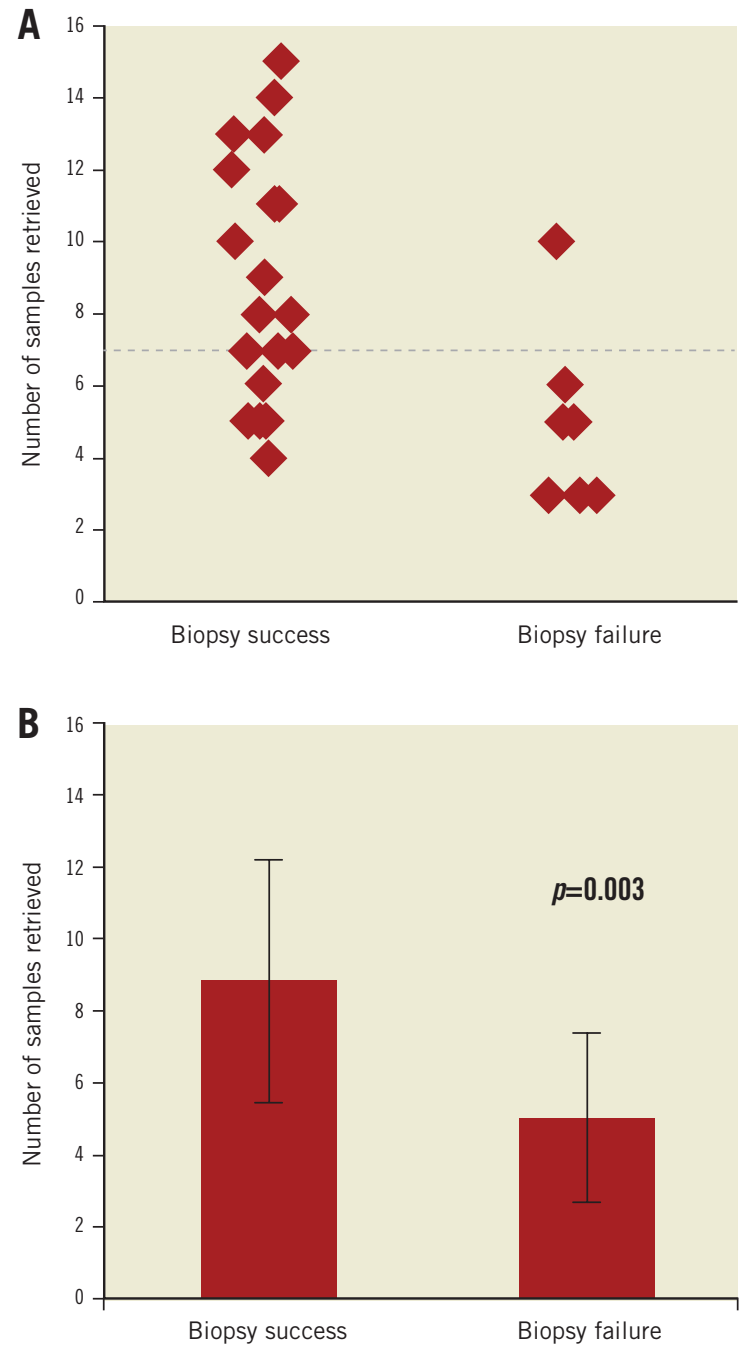

Figure 3. Biopsy outcomes in relation to the number of samples retrieved. A) Jitter plot showing the distribution of the number of samples retrieved during each procedure stratified by biopsy outcomes. The dashed red line is the median number of biopsies taken for the entire series. Sample numbers above the median significantly increased biopsy success. B) There was a significant difference between the average number of samples for biopsy success and biopsy failure.

Local anaesthesia was applied to the anterior chest wall. Care was taken to avoid the coronary arteries. A percutaneous transthoracic right ventricular biopsy with a Jamshidi needle was performed (Figure 5). A biopsy result of cardiac aspergillosis was subsequently rendered. No complications occurred.

\section{BIOPSY OUTCOMES}

Imaging with $\mathrm{CT}(76 \%)$, MR (55\%) and positron emission tomography (PET) (34\%) had been performed in the majority of patients prior to biopsy, without establishing a definite diagnosis. A positive TCB result was returned in $72 \%$ of patients. The TCB alone was the source of final diagnosis in 15 (52\%) patients. The final diagnosis was established by cardiac surgical excisional 

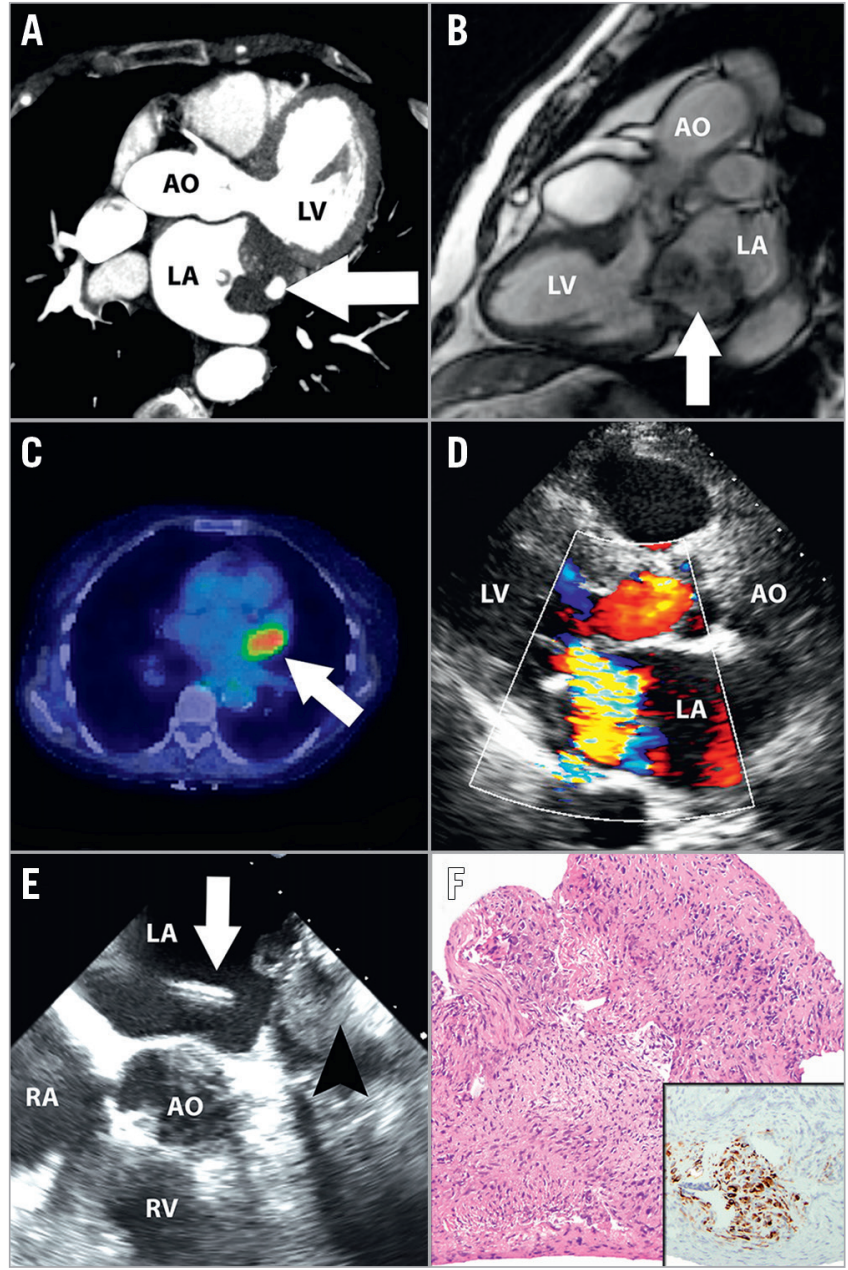

Figure 4. Transvenous transseptal biopsy of left atrial mass.

A) Cardiac CT showing dense left atrial mass (arrow) filling the left atrial appendage and abutting the left upper pulmonary vein.

$B$ ) Cardiac MR showing the heterogenous and multilobulated nature of the left atrial mass (arrow). C) Fusion PET scan showing avid FDG uptake by the left atrial mass (arrow). D) TTE showing the mass adjacent to the posterior mitral leaflet producing severe mitral regurgitation. E) TEE showing the transseptal puncture site with the sheath and bioptome (white arrow) approaching the mass (arrowhead). F) Photomicrograph of the biopsy specimen shows a malignant proliferation of spindled, hyperchromatic cells growing in vague fascicles (haematoxylin and eosin, 100x original magnification). Immunoperoxidase studies showed antigenic reactivity with desmin (inset, 100x original magnification), confirming a diagnosis of epithelioid leiomyosarcoma. CT: computed tomography; FDG: fluorodeoxyglucose; MR: magnetic resonance; PET: positron emission tomography; TEE: transoesophageal echocardiography; TTE: transthoracic echocardiography

biopsy in $38 \%$ and biopsy of extracardiac sites in the remaining $10 \%$. The most common diagnosis was sarcoma (24\%), including: angiosarcoma (4 cases), leiomyosarcoma (1 case), undifferentiated pleomorphic sarcoma (1 case) and endometrial stromal sarcoma (1 case). Myriad infectious, neoplastic, thrombotic and hamartomatous diseases were also seen (Table 3). TCB changed the plan of care in 52\% of patients overall (Figure 6). Eleven patients $(38 \%)$ who would have required excisional biopsy were able to avoid cardiovascular surgery entirely.

\section{Discussion}

To the best of our knowledge, this series is the sole systematic description of TCB for ICMs. We show that this procedure can be safely performed using echocardiographic guidance. It provides an accurate histopathologic diagnosis, altering clinical management in the majority of cases. Our analysis supports the notion that TCB may be used as a first-line approach for histopathologic diagnosis of ICM.

\section{CASE SELECTION}

The ideal clinical case for TCB would be an undiagnosed asymptomatic ICM where the histopathologic diagnosis cannot be established without resorting to cardiovascular surgery. Symptomatic masses may produce flow obstruction, valve damage, pulmonary embolisation and systemic embolisation. In such cases, cardiovascular surgery for mass removal would be indicated anyway and TCB is unlikely to provide additional diagnostic information.

Table 3. Final histopathologic diagnoses for intracardiac masses.

\begin{tabular}{|c|c|}
\hline Final mass histopathology & n (\%) \\
\hline Primary tumours & $10(34.5)$ \\
\hline Angiosarcoma & 4 \\
\hline Leiomyosarcoma & 1 \\
\hline Endometrial sarcoma & 1 \\
\hline Pleomorphic sarcoma & 1 \\
\hline Papillary fibroelastoma & 1 \\
\hline Intracardiac thymoma & 1 \\
\hline Intracardiac lipoma & 1 \\
\hline Metastatic tumours & $3(10.3)$ \\
\hline Renal cell carcinoma & 1 \\
\hline Squamous lung carcinoma & 1 \\
\hline Papillary thyroid carcinoma & 1 \\
\hline Lymphomas & $5(17.2)$ \\
\hline Diffuse large B cell & 4 \\
\hline Hodgkin's lymphoma & 1 \\
\hline Thrombotic & $3(10.3)$ \\
\hline Organised thrombus & 3 \\
\hline Infectious & $3(10.3)$ \\
\hline Candidiasis & 1 \\
\hline Aspergillosis & 2 \\
\hline Inflammatory & $3(10.3)$ \\
\hline Wegener's granulomatosis & 1 \\
\hline Granulomatous myocarditis & 2 \\
\hline Miscellaneous & $2(6.9)$ \\
\hline Rosai-Dorfman disease & 1 \\
\hline Myocardial hamartoma & 1 \\
\hline
\end{tabular}




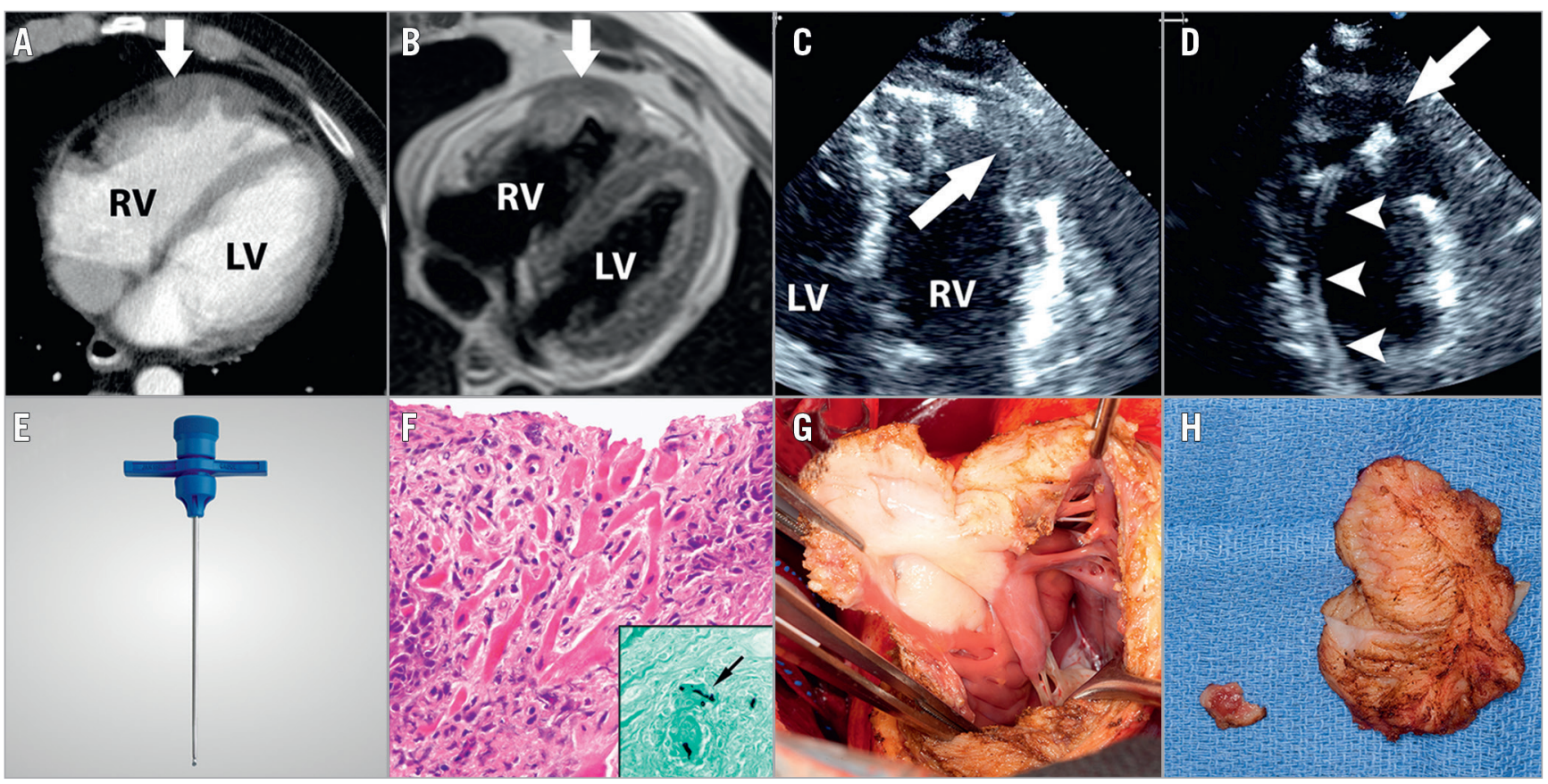

Figure 5. Percutaneous transthoracic biopsy of right ventricular mass. A) Cardiac CT showing the heterogenously enhancing mass in the anterior wall of the right ventricle (arrow). B) Cardiac MR showing transmural ventricular extension of the mass (arrow). C) Intraprocedural TTE showing the cavitating mass in the free wall of the right ventricle (arrow). D) Intraprocedural TTE showing the bioptome shaft in the right ventricle (arrowheads) and bioptome head (arrow) making contact with the mass. E) Representative image of a Jamshidi bone marrow biopsy needle used for percutaneous transthoracic biopsy of the right ventricular mass. F) Photomicrographs of the biopsy specimen show multiple fragments of dense, granulomatous inflammation involving the myocardium (haematoxylin and eosin, 400x original magnification). Grocott's methenamine silver stain highlights rare septate branching hyphae, morphologically consistent with Aspergillus species (400x original magnification). Infiltrating transmural mass seen in situ (G) and after excision by the cardiovascular surgical team (H). CT: computed tomography; MR: magnetic resonance; TEE: transoesophageal echocardiography; TTE: transthoracic echocardiography

Two exceptions to this rule might exist. The first would be a symptomatic ICM with significant extracardiac spread. Establishing a diagnosis of an incurable disease here could trigger palliation and spare the patient from undergoing cardiovascular surgery. In selected cases, the risk of morbidity and mortality from TCB of ICM may be lower than that for extracardiac sites such as the liver or kidneys. The second would be a surgically unresectable ICM localised to the heart. In addition to sparing the patient from undergoing futile surgery, establishing a diagnosis non-invasively could trigger therapy with antibiotics, anticoagulation, chemotherapy, radiotherapy, or perhaps an informed transition to palliative care.

\section{PROCEDURE PLANNING}

The uniform use of echocardiography in this series contributed to the high diagnostic yield from TCB. Visualisation with echocardiography is necessary to target the bioptome to the mass and to avoid

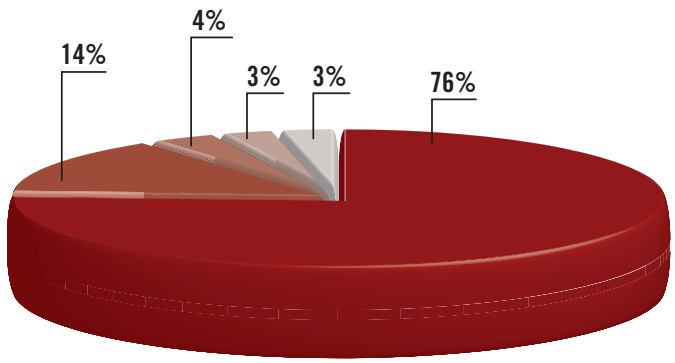

Pre

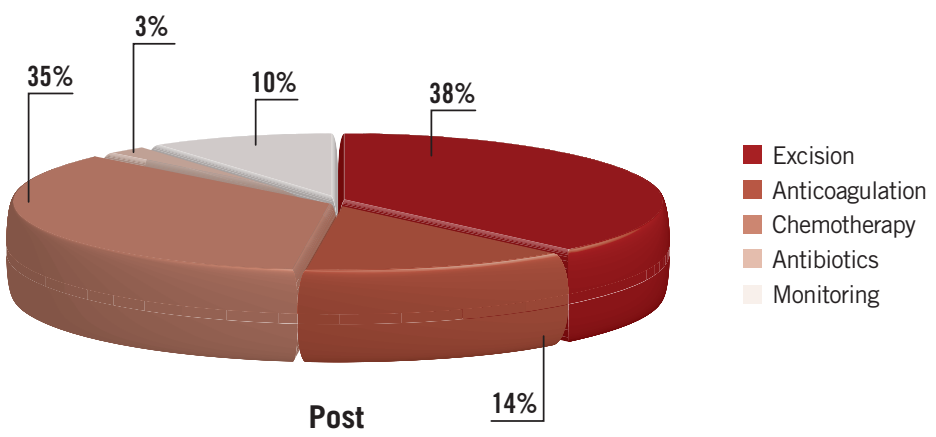

Figure 6. Clinical strategy before and after transcatheter biopsy. Pie charts showing clinical strategy for the entire series before and after transcatheter biopsy of intracardiac masses. There was a marked shift in management strategy in cases with biopsy success with a significant number of patients avoiding cardiovascular surgery. 
damaging native cardiac structures ${ }^{10}$. Depending on the mass location and imaging windows, either TTE or TEE could be employed. Moderate sedation and local anaesthesia should be used for TTEguided cases, but general anaesthesia and endotracheal intubation may be required for TEE-guided cases where the patient is unable to tolerate probe insertion awake. ICE may provide additional imaging guidance in cases where other echocardiographic windows are limited. Prior to the procedure, the platelet count and coagulation profile should be corrected to institutional invasive procedure standards. The masses in this series were located predominantly in rightsided chambers. In cases of multifocal locations, the right-sided lesions were biopsied. The approach to left-sided masses must be performed using a venous transseptal approach or arterial retroaortic approach and is inherently more complex. Either the internal jugular or common femoral approach can be used depending on operator preference. We recommend using the femoral approach when steerable sheaths or transseptal punctures are contemplated. Sheath length should be long enough to position the tip as close to the ICM as possible. This facilitates rapid exchange of the bioptome during multiple sampling attempts.

\section{BIOPSY TECHNIQUE}

We recommend initial use of the jaws-type bioptome for all ICMs, since this device is widely available and simple to use. The jaws should be opened before making contact with the mass and closed after advancing the bioptome into the mass. The sampling site should be definitively visualised using echo to avoid inadvertent biopsy of native cardiac structures. Since each additional sample provides significant incremental diagnostic value, operators should attempt to retrieve as many samples as possible. It is unclear if the individual biopsies should be taken from different locations on the mass to improve sampling, or if repeated biopsies at one site allow increased mass penetration on each subsequent pass. Many cardiac masses are encapsulated and the bioptome jaw may not engage the surface of the mass. When penetration is limited and tissue yield is poor, a transcatheter side-cutting biopsy needle can be used at the operator's discretion. There may be a role for immediate frozen section analysis to determine the adequacy of sampling during the procedure $^{11}$. The addition of a steerable sheath may improve bioptome access to masses that are relatively inaccessible.

\section{COMPLICATION RATES}

Ventricular tachycardia requiring immediate cardioversion was noted in two patients $(6.9 \%)$. This was triggered, in both cases, by bioptome contact with the ventricular wall. There were no vascular access complications, vasovagal reactions, cardiac perforation, pericardial tamponade, conduction disturbance, pulmonary embolus or systemic embolus. There was no procedure-related mortality. This highlights the safety profile of this procedure for right-sided masses. The global experience with transseptal antegrade or transaortic retrograde biopsy of left-sided masses is limited ${ }^{12}$. An individual risk benefit analysis should be conducted for the probability of mass embolisation versus the need to make a histopathologic diagnosis.
Because ICMs can be friable or have fragile pedicles, the theoretical risk of embolisation may be higher than that reported for endomyocardial biopsy. The use of cerebral embolic protection devices should be considered in high-risk cases. The one patient $(3.5 \%)$ in our series who had a percutaneous transseptal biopsy of a left atrial mass had no embolic complications. There remains a theoretical concern that TCB might embolise tumour fragments into the pulmonary or systemic circuits, possibly inciting haematogenous spread. There was incomplete follow-up in this series but, within these limits, no biopsy-induced metastases were noted.

\section{DIAGNOSTIC ACCURACY}

There was a positive biopsy yield in $72 \%$ of patients who underwent TCB. As shown in the regression models, each additional sample increased the likelihood of positive yield by $70 \%$, and retrieving more than seven samples provided a 16 -fold increase in the probability of diagnostic biopsy. While it could be argued that larger masses may have yielded more samples, we have shown that the relationship of sample number to positive yield is independent of mass size. It is possible that fewer samples were retrieved from masses located near sensitive structures and masses relatively inaccessible to the bioptome; these are some limitations of TCB for ICM. It may be that tissue characteristics of some masses make them more amenable to biopsy than others, but the myriad diagnoses encountered in this series show that TCB is versatile and can sample different types of ICM.

\section{CLINICAL UTILITY}

Overall, clinical management was altered in $52 \%$ of patients and in $71 \%$ of patients with biopsy success. The indications for cardiovascular surgery and excisional biopsy in the setting of cardiac masses include: 1) documentation of definite histopathologic diagnosis, and 2) correction of mass-related symptoms. Surgical excision may not be required in cases where the diagnosis can be demonstrated by TCB of ICM. This was true for 11 patients $(38 \%)$ in the series, who then received other histopathology-targeted therapies such as antibiotics, anticoagulation, chemotherapy, radiotherapy or even clinical monitoring. Patients in whom metastatic cancer is suspected and palliation is intended would benefit the most from avoiding cardiovascular surgery. Surgical resection becomes inevitable for symptomatic masses refractory to medical therapy. Preoperative planning can be improved by prior knowledge of mass histopathology. For example, it would be imperative to have complete resection and clean margins for primary neoplastic lesions, but this would not hold true for thrombus or hamartoma. Surgical excision may also be facilitated by neoadjuvant therapy directed against the histopathologic characteristics of the mass. Therefore, TCB for ICM has a role in both asymptomatic and symptomatic cases.

\section{Limitations}

The limitations of this study include its retrospective nature and small sample size. All biopsy procedures were performed at a single tertiary academic medical centre with significant referral bias. 
We do not have data on patients with ICM at our institute who were not referred for TCB. The possibility of selection bias exists with regard to lesion accessibility for transcatheter biopsy.

\section{Conclusions}

TCB can provide an accurate diagnosis for the majority of ICMs, especially for right-sided masses which are readily accessible by transcatheter devices. The complication rates are low in the hands of skilled operators guided by echocardiographic imaging. It provides clinically useful information that guides decision making in a significant proportion of patients. This makes it an excellent minimally invasive alternative to excisional biopsy via cardiovascular surgery, which may be associated with significant morbidity and possible mortality. TCB may be considered a potential firstline approach to the histopathologic diagnosis of ICMs.

\section{Impact on daily practice}

Transcatheter biopsy can provide a histopathologic diagnosis for intracardiac masses. The likelihood of diagnosis is increased with each additional sample. Cardiovascular surgery may not be required in cases where the diagnosis can be established by this technique. This should be considered a potential first-line approach to the diagnosis of intracardiac masses.

\section{Conflict of interest statement}

The authors have no conflicts of interest to declare.

\section{References}

1. Roberts WC. Primary and secondary neoplasms of the heart. Am J Cardiol. 1997;80:671-82.

2. Vander Salm TJ. Unusual primary tumors of the heart. Semin Thorac Cardiovasc Surg. 2000;12:89-100.
3. Peters PJ, Reinhardt S. The echocardiographic evaluation of intracardiac masses: a review. J Am Soc Echocardiogr. 2006;19: 230-40.

4. Rajiah P, Kanne JP, Kalahasti V, Schoenhagen P. Computed tomography of cardiac and pericardiac masses. J Cardiovasc Comput Tomogr. 2011;5:16-29.

5. Motwani M, Kidambi A, Herzog BA, Uddin A, Greenwood JP, Plein S. MR imaging of cardiac tumors and masses: a review of methods and clinical applications. Radiology. 2013;268:26-43.

6. Araoz PA, Eklund HE, Welch TJ, Breen JF. CT and MR imaging of primary cardiac malignancies. Radiographics. 1999;19: 1421-34.

7. Klarich KW, Enriquez-Sarano M, Gura GM, Edwards WD, Tajik AJ, Seward JB. Papillary fibroelastoma: echocardiographic characteristics for diagnosis and pathologic correlation. J Am Coll Cardiol. 1997;30:784-90.

8. Elbardissi AW, Dearani JA, Daly RC, Mullany CJ, Orszulak TA, Puga FJ, Schaff HV. Survival after resection of primary cardiac tumors: a 48-year experience. Circulation. 2008;118: S7-15.

9. Murphy MC, Sweeney MS, Putnam JB Jr, Walker WE, Frazier OH, Ott DA, Cooley DA. Surgical treatment of cardiac tumors: a 25-year experience. Ann Thorac Surg. 1990;49:612-7.

10. Satya K, Kalife G, Navarijo J, Rasekh A, Wilson JM. Transseptal biopsy of a left atrial mass with 3-dimensional transesophageal echocardiographic guidance. Tex Heart Inst J. 2012;39:707-10.

11. Winters GL, Hauptman PJ, Jarcho JA, Schoen FJ. Immediate evaluation of endomyocardial biopsies for clinically suspected rejection after heart transplantation. Circulation. 1994;89: 2079-84.

12. Jackson CE, Gardner RS, Connelly DT. A novel approach for a novel combination: a trans-septal biopsy of left atrial mass in recurrent phyllodes tumour. Eur J Echocardiogr. 2009;10:171-2. 